\title{
La rencontre de Verdi et de Shakespeare : le traitement de la voix
}

Gilles de Van

\section{(2) OpenEdition}

\section{Journals}

Édition électronique

URL : http://journals.openedition.org/shakespeare/355

DOI : 10.4000/shakespeare.355

ISSN : 2271-6424

Éditeur

Société Française Shakespeare

\section{Édition imprimée}

Date de publication : 1 novembre 1999

Pagination : 109-118

ISBN : 2-84269-331-0

\section{Référence électronique}

Gilles de Van, "La rencontre de Verdi et de Shakespeare : le traitement de la voix », Actes des congrès de la Société française Shakespeare [En ligne], 17 | 1999, mis en ligne le 01 novembre 2007, consulté le 20 avril 2019. URL : http://journals.openedition.org/shakespeare/355 ; DOI : 10.4000/

shakespeare.355 


\section{S H A K E S P E A R E \\ \& $\quad$ L $A \quad$ V O I X}

Société Française Shakespeare

Actes du Congrès de 1999

米米

Textes réunis et présentés par

Patricia DORVAL

publiés sous la direction de

Jean-Marie MAGUIN 


\section{LA RE NCONTRE DE VERDI ET DE SHAKESPEARE : LE TRAITEMENT DE L A VO I X}

Avant d'en venir au problème de la voix qui intéresse directement ce colloque, il me semble utile de commencer par quelques rapides généralités qui situent le cadre d'ensemble de ma communication.

Verdi a mis en musique trois pièces de Shakespeare : Macbeth en 1847, opéra révisé en 1865 ; Othello en 1887 et Falstaff en 1893. Il a longuement travaillé sur une adaptation de King Lear entre 1845 et 1865 (on ne sait pas très bien quand il a abandonné le projet). Il a envisagé d'autres projets shakespeariens mais sans leur donner une réalisation musicale concrète : Hamlet et La tempête et enfin Roméo et Juliette.

Cette collaboration est importante mais là n'est pas l'essentiel. L'essentiel est ailleurs : de manière générale, les dramaturges, de quelque origine qu'ils soient, sont considérés par les musiciens et les librettistes comme des pourvoyeurs d'histoires, de «scénarios». Ils fournissent des histoires que l'on adapte en fonction des conventions lyriques de l'époque et des goûts du compositeur. Il me semble que Verdi est le premier musicien à estimer - et à proclamer nettement - que par-delà leurs histoires, certains auteurs sont des dramaturges qui ont une pensée sur le théâtre, sur la vie et sur les grands problèmes de la fiction. Schiller et Hugo sont pour lui des dramaturges, mais son point de référence absolu et constant est Shakespeare qu'il appelle familièrement «papa» et qu'il qualifie de 
«grand maître du cœur humain» ${ }^{1}$. Autrement dit, pour notre musicien, Shakespeare est un maître de vie et de pensée.

Cette vénération s'accompagne d'une influence directe sur certains points. Je n'en citerai que deux et tous deux concernent Macbeth ; c'est un opéra de jeunesse car Verdi, né en 1813 , a commencé sa carrière en 1839 ; son premier grand succès date de 1842, tandis que la première version de Macbeth date de 1847 :

1) la mise en scène: les compositeurs italiens sont dans l'ensemble des praticiens, soucieux de la réalisation visuelle des spectacles qu'ils écrivent. Verdi ne fait pas exception à la règle mais, que je sache, c'est pour Macbeth que Verdi surveille avec une attention particulière la réalisation visuelle, les emplacements, les accessoires scéniques, les étoffes, les maquillages, etc. Cette minutie est évidente pour certaines scènes comme la grande scène des apparitions (acte III de l'opéra) ou pour la scène du banquet où le spectre de Banquo occupe la place qui lui revenait vivant (acte II). La peur de l'échec ou du ridicule compte pour beaucoup car la séduction de l'opéra est fragile et la moindre bévue la détruit; mais il faut aussi songer au caractère insolite, dans l'histoire lyrique, des scènes fantastiques qui doivent être préparées avec la plus grande vigilance.

2) le langage : le personnage collectif des sorcières ${ }^{2}$ appelle un style lui aussi tout à fait nouveau dans la langue stéréotypée et conventionnelle de l'opéra. Verdi, qui lisait Shakespeare à travers l'interprétation de Schlegel, voulait que les sorcières fussent à la fois triviales, grotesques et sublimes. Son librettiste F.M. Piave ne réussissait pas à trouver le ton juste car, dans l'univers lyrique de ces années, il était presque impossible de concevoir un «personnage» à la fois noble, sérieux et cocasse. Pour vous donner une petite idée des difficultés du librettiste, je citerai une phrase de Verdi à Piave, pendant la phase préparatoire de la première version: «Bien que ce chœur de sorcières ne $m$ 'ait pas plu du tout, j'ai toutefois ri de bon coeur en voyant que tu avais mis en tête de la scène "sorcières en habit de cérémonie"» $\left(10\right.$ déc. 1846) ${ }^{3}$. On aura compris que le pauvre Piave était loin du compte, et d'ailleurs le compositeur s'adressa à un autre poète pour ces passages.

Voilà donc, pour une œuvre de jeunesse dont l'importance dans le cheminement de Verdi est unanimement reconnue, deux aspects qu'il suit de très près et qui sont tous deux liés à Shakespeare. Je signale du reste en passant que nous avons abondance de lettres sur la genèse de cet opéra, les Américains ayant réuni toute la documentation disponible dans un ouvrage qui est cité à la note 3 . 
J'en viens au sujet de mon exposé. Quelle est l'incidence de Shakespeare dans le rapport de Verdi à la voix ? La réponse est simple: chaque fois que Verdi met en question le personnage traditionnel et donc l'élaboration du style vocal qui lui convient, il le fait à l'occasion de la rencontre avec un dramaturge ; ce peut être Victor Hugo (c'est très clair avec Rigoletto par exemple), mais de manière plus fréquente et radicale, c'est Shakespeare.

Pour bien me faire comprendre, je vais indiquer un exemple concret et citer une lettre célèbre qu'en novembre 1848 , donc après la création de la première version de Macbeth, Verdi adressa de Paris à Cammarano, un librettiste qui, comme c'était souvent le cas, faisait fonction de directeur de la scène au théâtre San Carlo de Naples; il y récusait comme interprète du rôle féminin Eugenia Tadolini, grand soprano dramatique de l'époque que par ailleurs il admirait beaucoup ; je précise enfin que cette lettre fut inutile car la Tadolini joua le rôle :

Je sais que vous préparez Macbeth et comme c'est un opéra auquel je m'intéresse plus particulièrement, permettez-moi de vous en dire quelques mots. On a confié à la Tadolini le rôle de Lady Macbeth et je suis surpris qu'elle ait accepté de jouer ce rôle. Vous savez toute l'estime que $\mathrm{j}$ 'ai pour la Tadolini et elle-même le sait, mais dans notre intérêt je crois nécessaires ces quelques réflexions. La Tadolini a une figure belle et bonne, et je voudrais que Lady fût laide et méchante. La Tadolini chante à la perfection ; et je voudrais que Lady ne chantât point. La Tadolini a une voix étonnante, claire, limpide, puissante; et je voudrais pour Lady une voix âpre, étouffée, sombre. La voix de la Tadolini a quelque chose d'angélique; je voudrais que la voix de Lady eût quelque chose de diabolique 4 .

Pour que le sens de cette lettre apparaisse pleinement, deux observations sont nécessaires: Verdi se développe dans une civilisation lyrique qui est celle du bel canto. Les aspects techniques du bel canto (virtuosité, trilles, gammes) sont bien connus, mais leur fondement dramaturgique n'est pas toujours aussi clair. On peut le préciser avec un écrit d'un ami de Rossini qui a essayé de transcrire ses idées esthétiques : 
La musique produit des effets merveilleux quand elle accompagne l'art dramatique, quand l'expression idéale de la musique s'associe à l'expression vraie de la poésie, et à l'expression imitative de la peinture. Alors, tandis que les mots et les gestes expriment les détails les plus menus et concrets des passions (affetti), la musique se propose un but plus élevé, plus ample, plus abstrait ${ }^{5}$.

Rossini décrit un processus d'idéalisation qui est confié à la musique et au chant, et qui me semble être la justification dramaturgique du bel canto. Ce processus est fortement entamé par la pratique de musiciens comme Donizetti qui perturbent l'harmonie de ce monde idéal au nom de la violence des passions. C'est la dernière étape de ce processus qui est niée par Verdi au profit de la vérité dramatique de Lady; autrement dit, Lady n'est pas compatible avec le monde du bel canto traditionnel et il faut inventer un chant autre.

Ma deuxième observation porte justement sur ce chant autre : Verdi s'est expliqué très clairement dans les documents qui accompagnent la genèse de l'œuvre. On est frappé, dans la partition, par le nombre d'indications concernant le registre vocal qui doit être utilisé et les changements de registre vocal. Voyez par exemple les indications de l'auteur dans le duo Banco-Macbeth qui suit la triple salutation des sorcières comme seigneur de Glamis, seigneur de Cawdor et roi d'Écosse, et l'arrivée des messagers qui annoncent l'exécution du seigneur de Cawdor et l'attribution de ce titre à Macbeth : «fra sé, sottovoce, quasi con ispavento, con esclamazione, esclamando, cupo, a voce aperta» (en aparté, à voix basse, presque avec épouvante, exclamatif, en s'exclamant, sombre, à pleine voix). Vous remarquerez que l'utilisation de la voix pleine («a voce spiegata, voce piena») est strictement contrôlée et que Verdi signale les passages où la voix doit être retenue et ceux où elle peut se déployer. Encore plus frappante l'indication au début du grand duo entre Macbeth et sa femme, au cours duquel le général tuera le roi Duncan : «tout ce duo devra être dit par les chanteurs d'une voix basse et sombre («sotto voce») à l'exception de quelques phrases où sera indiqué d'une voix pleine ${ }^{6}$ («a voce spiegata»)».

J'ajoute encore quelques citations sur un plan plus général : dans la lettre où il discute le choix de la Tadolini, Verdi ajoute à propos des deux morceaux qui lui semblent fondamentaux dans l'œuvre, le grand duo Macbeth-Lady Macbeth qui précède le 
meurtre de Duncan et la scène du somnambulisme : «[...] et ces morceaux ne doivent absolument pas être chantés : il faut les agir et les déclamer d'une voix bien sombre et voilée; sans cela, l'effet ne peut être atteint». Un témoignage de $\mathrm{E}$. Muzio, le seul élève de Verdi et souvent le fidèle interprète de ses pensées, date de la composition de Macbeth :

Aucun acteur ne peut, actuellement en Italie, faire mieux Macbeth que Varesi, en raison de sa façon de chanter, de son intelligence, et même de sa silhouette petite et vilaine. Vous me direz peut-être qu'il ne chante pas très juste, cela ne fait rien car le rôle devrait être presque entièrement déclamé et pour cela il est très bon. 7

Et enfin, une citation tirée des mémoires de la créatrice du rôle de Lady, Marianna Barbieri Nini : «Et le duo avec le baryton qui commence Fatal mia donna, un murmure, vous croirez que j'exagère mais il fut répété plus de cent cinquante fois : pour obtenir, disait le maestro, qu'il fût plus discouru que chanté» ${ }^{8}$.

Les choix stratégiques de Verdi sont donc clairs. On peut longuement s'interroger sur les raisons de ces choix; j'ai déjà cité une raison négative : bloquer le processus de la sublimation par le chant. Le compositeur suggère également une puissance démoniaque du personnage, laid et maléfique, dont la voix âpre et étouffée est une digne métaphore. La violence et l'impétuosité de Lady, voire son érotisme dévoyé, ne me paraissent pas être en cause car on compte, bien avant Lady, des femmes énergiques, violentes, passionnées, comme les reines d'Angleterre de Donizetti ou Lucrèce Borgia du même Donizetti. On peut encore invoquer le caractère résolument nocturne, texte et musique, de la partition dont le traitement vocal serait un équivalent (sotto voce = à voix basse). On peut enfin, et c'est ce qui ressort nettement d'interprétations récentes, estimer que le «sotto voce» est l'équivalent vocal d'un rapport trouble de soi à soi, d'un décalage entre une conscience claire et une zone plus sombre dont le personnage n'est qu'en partie conscient ou bien qu'il hésite à formuler «à la lumière du jour».

Un exemple simple et complet de la perspective adoptée par le compositeur est offert par l'air du somnambulisme chanté par Maria Callas. Il faut en fait écouter la scène tout entière car Verdi l'a conçue comme un tout indissociable (en effet elle s'intitule «gran scena» et non pas «scena ed aria»), où ce qui précède l'air 
compte autant que l'air proprement dit. Ce dernier repose sur une ligne mélodique admirable et très bien équilibrée mais constamment morcelée comme l'est d'ailleurs le texte. Vous noterez en outre la diversité des registres vocaux dont use Callas, ce qui permet de comprendre ce que Verdi entendait par mal chanter : non point ne pas savoir chanter mais chanter assez parfaitement pour évoquer un chant désordonné et perturbé. J'ajoute enfin que cet air est ponctué d'interventions du médecin et de la dame de compagnie qui accompagnent l'agonie de la protagoniste.

$$
* * *
$$

Faisons maintenant un saut de quarante ans et arrivons à Otello qui date de $1887^{9}$. Comme je l'ai fait pour Macbeth, je partirai d'une citation qui me semble exemplaire et qui illustre mon discours. En 1887, Verdi a beaucoup de mal pour trouver une bonne Desdémone et il explique sa conception à son éditeur Ricordi :

Desdémone est un rôle où le fil, la ligne mélodique ne cesse jamais de la première à la dernière note. De même que Iago ne doit que déclamer et ricaner ${ }^{10}$; de même qu'Otello, tantôt guerrier, tantôt amant passionné, tantôt prostré jusqu'à la lâcheté, tantôt féroce comme un sauvage, doit chanter et hurler; de même Desdémone doit chanter toujours, toujours. ${ }^{11}$

Cette citation fixe de manière très claire ce que j'ai appelé les choix stratégiques de Verdi : un rôle constamment lyrique pour Desdémone que Verdi voit comme un de ces personnages shakespeariens qu'il définit des anges. Iago doit déclamer et ricaner, donc chanter le moins possible ; et Otello doit cumuler tous les styles vocaux, chant, déclamation, cri. Comme ouvre, Otello mélange tous les styles, air, récitatif, arioso et registres intermédiaires, en sorte qu'on passe insensiblement d'une écriture à l'autre.

Ce que je voudrais toutefois souligner, dans la perspective du thème principal de ce colloque, c'est la crise du ténor qui apparaît pleinement avec le personnage d'Otello, donc par l'intermédiaire d'un personnage shakespearien. Traditionnellement, et ce jusqu'à Radamès (Aida 1871) ou jusqu'à Adorno (Simon Boccanegra deuxième version 1881), le ténor est un personnage monolithique, 
taillé dans le roc, fidèle à certaines valeurs comme la loyauté, l'honneur, le courage. Le fait qu'il succombe la plupart du temps n'entame en rien sa cohérence morale : il a l'éclat du héros et aussi ses faiblesses; il incarne superbement ce que Tennyson appelait «the divine stupidity» du héros. Alors que les grandes héroïnes verdiennes connaissent très tôt l'incertitude, la contradiction, la mobilité intérieure, le héros-ténor garde sa fixité statuaire jusque très tard et ce n'est qu'avec Otello qu'il entre en crise.

Je ne vais pas décrire à des shakespeariens les raisons du poignant effondrement de ce personnage qui apparaît au départ auréolé de grandeur et de gloire. Je le prends comme une donnée de fait qui met en question non seulement un personnage mais toute une dramaturgie fondée sur la clarté et la cohérence des attitudes. Or, il faut traduire vocalement cette progressive destruction du héros à laquelle participent et le travail de sape de Iago et la vulnérabilité d'Otello. On ne peut qu'admirer l'extraordinaire mobilité de la ligne vocale du protagoniste qui oscille entre la mélodie organisée et le cri : c'est que tous les registres de chant doivent être présents pour suggérer et la noblesse du personnage et la catastrophe qui le mine.

Un bon exemple de ce traitement de la voix pour le ténor est le troisième duo entre Otello et Desdémone de l'acte III, qui est immédiatement suivi de son grand monologue. J'en rappelle les principaux moments. I : le ton est au départ imposé par Desdémone qui est, vous le savez, une fille de la bonne société vénitienne: «Que Dieu te tienne en joie, ô maître de mon âme» ${ }^{12}$; il est donc élégant et mondain. Desdémone chante, car elle doit chanter toujours, et Otello la mime, non sans ironie. II : l'allusion de Desdémone à Cassio brise instantanément cette aura d'élégance un peu frelatée et instaure à l'orchestre un discours fiévreux qui justifie que la conversation tombe sur le mouchoir. La scène est agitée et Otello déclame avec fougue, tout en rappelant avec solennité l'origine magique du mouchoir. III : dans la troisième section, Desdémone tente de reprendre le contrôle de la situation en reparlant de Cassio, c'est-à-dire en revenant à son style élégant et mondain : «Tout cela n'est qu'un jeu rusé, pour détourner ma requête et le voeu de Cassio». Mais c'est trop tard, Otello a perdu toute mesure, il demande le mouchoir et accuse d'impureté sa femme, jusqu'à ce que Desdémone prenne conscience de la violence de son époux. IV : alors elle plaide douloureusement son innocence et elle chante dans un tout autre style d'une grande pureté lyrique, débarrassé de toute mondanité : «Vois ma douleur immense, vois mon immense angoisse - et mes premières larmes». 
$\mathrm{V}$ : la section suivante est très agitée car Otello ne peut plus se contrôler. VI : mais il faut terminer le duo et Verdi reprend le motif initial que le protagoniste va déformer sur le ton de l'ironie la plus amère. Il y a en quelque sorte deux fins pour ce duo : formellement et vocalement, le duo se termine par la reprise du motif initial; l'orchestre continue par un interlude violent au cours duquel Otello passe de la fureur à la prostration. C'est dans cet état d'esprit qu'il entame son grand monologue : «Dieu ! - Tu pouvais m'infliger tous les maux / De la misère et de la honte...». Déclamation presque recto tono qui peu à peu se transforme en chant lyrique.

Ce long passage offre un exemple des différents styles vocaux du personnage : le chant parodique (quand il imite sa femme), la proclamation solennelle ou courroucée qui aboutit au cri, au hurlement, la déclamation prostrée, monocorde, et enfin le lyrisme propre à Otello qui est celui de la nostalgie. Face à lui, Desdémone est fidèle à l'image que Verdi s'en est faite : chanter, toujours chanter. On peut dès lors poser la question : existe-t-il dans le théâtre de Verdi un personnage autre qu'Otello, donc un personnage shakespearien, qui est assez complexe pour contraindre le musicien à une telle diversification de styles vocaux ?

Je conclurai comme j'ai commencé : le parcours de Verdi en cinquante ans de carrière est considérable dans tous les domaines (vocal, formel, dramatique). Ces changements ne résultent pas d'une théorie radicale qui aurait été posée à un moment donné de sa carrière puis appliquée rigoureusement, mais d'une navigation à vue qui profite de toutes les occasions qui lui sont offertes. Dans ce cheminement, c'est clairement non sur des théories mais sur des hommes qu'il s'est appuyé ; dans presque tous les cas, ces hommes n'étaient pas des musiciens mais des dramaturges. Parmi ces modèles durables ou éphémères, il me semble évident que Shakespeare a constitué à la fois une source d'inspiration et de réflexion permanente, à nulle autre comparable.

Gilles DE VAN 


\section{NOT E S}

${ }^{1}$ G. Verdi, I Copialettere di Giuseppe Verdi, a cura di G. Cesari, et A. Luzio, Milan, 1913 (Reprint Bologne, Forni, 1979), p. 276 \& 624.

${ }^{2}$ Elles sont trois chez Shakespeare et deviennent chez Verdi un chœur comprenant trois groupes de sopranos; le compositeur souhaitait $6 \times 3$ chanteuses.

${ }^{3}$ Verdi's Macbeth, a Sourcebook, ed. D. Rosen \& A. Porter, New York, Norton, 1984, p. 21.

${ }^{4}$ Macbeth sourcebook, op. cit., p. 66.

${ }^{5}$ Luigi Rognoni, Gioacchino Rossini, Turin, ERI, 1968, p. 379.

${ }^{6} \mathrm{~L}$ 'expression en italiques est de Verdi.

${ }^{7}$ Giuseppe Verdi nelle lettere di Emanuele Muzio ad Antonio Barezzi, a cura di L.A. Garibaldi, Milan, Treves, 1931, p. 261.

${ }^{8}$ Interviste $e$ incontri con Verdi, a cura di M. Conati, Milan, Il Formichiere, 1980, p. 25.

${ }^{9}$ Je précise qu'en passant à l'opéra, Othello devient Otello!

${ }^{10}$ En français dans le texte.

${ }^{11}$ Franco Abbiati, Giuseppe Verdi, Milan, Ricordi, 1959, IV, p. 337.

${ }^{12}$ Comme chacun sait, le texte italien de l'opéra est de Arrigo Boito. Mais ce même Boito est également l'auteur, avec C. Du Locle, de la version française (à chanter), et c'est elle que je cite. 